\title{
Comparison of the Effects of Dialysis Methods (Haemodialysis vs Peritoneal Dialysis) on Diastolic Left Ventricular Function
}

Dialysis methods and diastolic function

Fedoua Ellouali ${ }^{1,3}$ MD, Fatimazahra Berkchi ${ }^{2}$ MD, Rabia Bayahia ${ }^{2}$ MD, Loubna Benamar ${ }^{2} \mathrm{MD}$, Mohammed Cherti ${ }^{1} \mathrm{MD}$.

${ }^{1}$ Cardiology "B" department, Ibn Sina Hospital, Mohammed V University, Souissi, Rabat, Morocco

${ }^{2}$ Nephrology and Dialysis Center, Ibn Sina Hospital. Mohammed V University, Souissi, Rabat, Morocco

${ }^{3}$ Laboratory of Biostatistics, Clinical Research and Epidemiology, Faculty of Medicine and Pharmacy. Mohammed V University, Souissi, Rabat, Morocco.

Corresponding Author: ELLOUALI Fedoua, MD

Secteur 16 bloc I num 8, Hay Riad, Rabat, Morocco, 10100

E-mail: f.ellouali@hotmail.com

Phone number: 00212663507524

Fax number: 00212537727263 


\begin{abstract}
Introduction. In patients undergoing chronic dialysis, several factors appear to influence the occurrence of cardiac abnormalities. The aim of our study was to evaluate the effects of two different methods of renal replacement therapy (chronic haemodialysis (HD) and continuous ambulatory peritoneal dialysis (CAPD)) on left ventricular (LV) diastolic function.
\end{abstract}

Patients and methods. We enrolled 61 patients: 21 patients on CAPD, and 42 age- and gender-matched patients on HD; 35 patients were men (55.6\%). Median of age was 46.4[3557] years. The median duration of renal replacement therapy was 3[2-5] years.

Results. The two groups (HD vs PD) were similar concerning body mass index, dialysis duration and cardiovascular risk factors. The comparison of echocardiographic parameters showed statistically significant differences between two groups, regarding the presence of calcification, cardiac effusion, severely abnormal LVH and E/e' define all abbreviations $>13$ $\left(p=0.001, p=0.003, p=0.02, p=0.004\right.$, respectively). In multivariate analysis, an $E / e^{\prime}>13$ was 5.8 times higher commoner? in PD group (CI [1.3-25.5], p=0.002).

Conclusion. The method of dialysis seems to influence LV diastolic function. We observed a higher prevalence of diastolic LV dysfunction in the PD group. Echocardiographic follow up is essential as this could improve the management of cardiovascular complications in dialysis patients.

KEY WORDS: Diastolic Function, Echocardiography, End Stage Renal Disease, Haemodialysis, Left Ventricular Hypertrophy, Peritoneal Dialysis 


\section{INTRODUCTION}

Echocardiography is the most useful imaging technique for initial cardiac assessment, enabling detailed examination of the main cardiac structures and effective assessment of the left ventricular (LV) mass and changes in ventricular function [1]. Indeed, in patients undergoing chronic dialysis, left ventricular hypertrophy (LVH) and cardiac geometry influence LV dysfunction [2]. Several factors appear to influence the occurrence of cardiac abnormalities. Whether haemodialysis (HD) or peritoneal dialysis (PD) has a different impact on echocardiographic parameters has been previously investigated, but the results are heterogeneous and contradictory.

The aim of our study was to evaluate the effects of two different methods of renal replacement therapy (chronic HD and continuous ambulatory peritoneal dialysis (CAPD)) on echocardiographic parameters.

\section{PATIENTS AND METHODS}

\section{Patients}

We enrolled 63 patients; 21 patients on CAPD, and 42 age- and gender-matched patients on HD after obtaining informed consent.

35 patients were men $(55.6 \%)$. The median of age was $46.4[35-57]$ years. The median of duration of renal replacement therapy was $3[2-5]$ years.

Haemodialysis was performed three times a week for $4 \mathrm{~h}$. Echocardiographic parameters were measured within $2 \mathrm{~h}$ after a dialysis session_or peritoneal exchange.

\section{Inclusion criteria}

We included patients on renal therapy replacement for $>6$ months, who had an adequate echocardiographic examination. Would this introduce selection bias if echocardiographic examination was not routinely performed in all patients? 


\section{Exclusion criteria}

We excluded patients with severe anaemia, uncontrolled hypertension, diabetes, rhythm or conduction abnormality, valvular heart disease, past history of heart failure or unstable angina.

\section{Methods}

\section{Therapeutic modalities}

CAPD consists of 3 to 4 exchanges/day. All haemodialysis patients had a radial arteriovenous fistula. Haemodialysis was carried out three times a week for $4 \mathrm{~h}$ with standard bicarbonate dialysis.

\section{Clinical data}

Baseline characteristics were collected: age, gender, dialysis duration (in years), hypertension, hyperlipidaemia and smoking.

Hypertension was defined as systolic blood pressure (BP) $\geq 140 \mathrm{mmHg}$, diastolic BP $\geq 90$ $\mathrm{mmHg}$ or the use of antihypertensive medication. For hypertensive patients, the strict control of BP was required and treatment with renin-angiotensin-system inhibitors was introduced.

Hyperlipidaemia was defined as total cholesterol $\geq 200 \mathrm{mg} / \mathrm{dL}$, low-density lipoprotein cholesterol (LDL-C) $\geq 130 \mathrm{mg} / \mathrm{dL}$ or the use of lipid-lowering medication.

\section{Biological data}

Routine laboratory methods were used to measure biochemical parameters: haemoglobin, Creactive protein (CRP), phosphorus, calcium, rate of intact parathyroid hormone (iPTH). Residual renal function (RRF) was estimated by calculating glomerular filtration rate (GFR). GFR was calculated ref according to the formula: GFR $=U_{\text {vol }} / t \times\left\{U_{\text {ureal }}\left(\right.\right.$ Pre $_{\text {Urea }}+$ Post $\left.\left._{\text {Urea }}\right) / 2\right]+U_{\text {creat }} /\left[\left(\right.\right.$ Pre $_{\text {Creat }}+$ Post $\left.\left.\left._{\text {Creat }}\right) / 2\right]\right\} \quad$ calcuSA (SA: surface area in $\mathrm{m}^{2}, \mathrm{t}$ : duration of collection between dialyses in minutes $\mathrm{U}_{\mathrm{vol}}$ : urine collection volume in $\mathrm{mL}$, Pre $\mathrm{Urea}_{\text {rea }}$ and 
Pre $_{\text {Creat: }}$ pre-dialysis urea and creatinine concentration in blood samples at the end of the

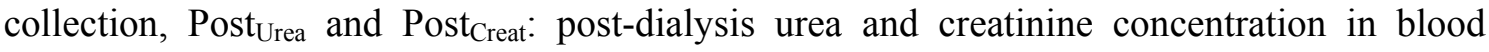
samples at the beginning of collection, and : urea and creatinine urine concentrations) [3]. The surface area (SA) was estimated by the Mosteller formula: $\mathrm{SA}=\sqrt{\mathrm{L} \times \mathrm{M} / 3600}$ (SA in $\mathrm{m}^{2}$, weight in $\mathrm{Kg}$, height in $\left.\mathrm{cm}\right)$. ref

Clinical and laboratory criteria for adequate nutrition were an albumin $\geq 35 \mathrm{~g} / 1$ and a body mass index $(\mathrm{BMI}) \geq 21 \mathrm{~kg} / \mathrm{m}^{2}$. Anaemia was defined as haemoglobin $<11 \mathrm{~g} / \mathrm{dl}$.

\section{Echocardiographic data}

Echocardiography was achieved by a GE, define all abbreviations Vivid 7 ultrasound system. The same cardiologist performed all echocardiographic examinations. The patients were examined in the left lateral decubitus position and data was collected in standard parasternal long-axis, short-axis, and apical views. Measurements were acquired by averaging three cardiac cycles.

Left ventricular hypertrophy is defined by an increase in LV mass (LVM) index $>95 \mathrm{~g} / \mathrm{m}^{2}$ in women and $>115 \mathrm{~g} / \mathrm{m}^{2}$ in men. LVM was considered as severely abnormal if LVM $\geq 122$ $\mathrm{g} / \mathrm{m}^{2}$ in women and $\geq 149 \mathrm{~g} / \mathrm{m}^{2}$ in men.

The following anatomically validated equation was used to estimate LVM: LVM = $0.8[1.04($ IVS + LVIDd + LVPW)3 - LVIDd3 $]+0.6$; where IVS is the diastolic septal thickness, LVIDd is the diastolic dimension of the LV cavity and LVPW is the diastolic thickness of the posterior wall.

Teicholz's and Simpson's methods were used to evaluate LV ejection fraction. Echocardiographic maximum left atrial volume was measured from apical 4- and 2-chamber views by biplane area-length $[4,5]$.

The values of the E wave and A wave of mitral flow were collected (on apical 4-chamber view, a $3 \mathrm{~mm}$, at the level of the basal mitral annulus). Tissue Doppler velocities of 
longitudinal mitral annular motion were measured in median and lateral segment of the basal mitral annulus (on apical 4-chamber view) and the mean e' (early diastolic velocity) was used to estimate $\mathrm{E} / \mathrm{e}$.

Peak systolic velocity (S), early (e') diastolic velocities were assessed for right and left annular side, successively.

\section{Statistical data}

Continuous variables are expressed as mean \pm standard deviation (or median with interquartile range (IQR) when the distribution is non-normal). Qualitative variables were analysed using the Chi2 test (or the Fisher exact test according to the number of cases), and quantitative variables were analysed using the $t$ test (or a nonparametric test according to the normality of distribution). Statistical significance was defined as $\mathrm{P}<0.05$ two-sided? Statistical analysis was performed using SPSS for Windows, version 11.0.

\section{RESULTS}

The mean body weight was $66 \pm 14.2 \mathrm{~kg}$. Hypertension was noted in $50.8 \%$ (31 cases). Antihypertensive drugs were similarly used in the two groups (figure 1). Nine patients $(14.8 \%)$ were smokers, and 21 patients (33.3 \%) had dyslipidaemia. The two groups (HD vs PD) were similar concerning body mass index, dialysis duration, residual renal function, anaemia and cardiovascular risk factors. Comparison of biological characteristics showed no statistically significant difference between the two groups. Data is reported in Table 1. In our study, LVH was noted in 28 patients (44.4\%) and LVM was considered as severely abnormal in 19 patients $(20.2 \%)$. There was no statistically significant difference between the two groups concerning $\mathrm{LVH}(\mathrm{p}=0.45)$ but severely abnormal LVH was more frequent in PD group (23.8\% in HD group vs $42.9 \%$ in PD group, $\mathrm{p}=0.02)$. There was a significant difference between the two groups concerning the presence of calcification, the presence of cardiac 
effusion and $\mathrm{E} / \mathrm{e}^{\prime}>13(\mathrm{p}=0.001, \mathrm{p}=0.003, \mathrm{p}=0.004$, respectively). The comparison of echocardiographic parameters is shown in tables 2 and 3. Multivariate logistic regression was used to evaluate the association of each significant echocardiographic parameters with dialysis methods. All significant variables were considered candidate confounders. In this regression model, and after adjustment for confounders, an E/e'>13 was 5.8 times higher commoner? in PD group (CI [1.3-25.5], $\mathrm{p}=0.002$ ) (figure 2).

\section{DISCUSSION}

\section{Is it relevant to measure diastolic function of the $\mathrm{LV}$ ?}

Mortality is known to increase with LV diastolic dysfunction [8]

Hsiao and al showed that in the uraemic population, LV dysfunction, LVM index and a high post-dialytic E/e' are prognostic of major events [6]. In the same way, in a study by Kim and al, diastolic dysfunction was a significant marker of rapid decline in residual renal function and the occurrence of cardiovascular events in patients placed in a peritoneal dialysis program [7].

\section{What are the methods of evaluation of diastolic function?}

In CKD (chronic kidney disease) patients, TDI is more sensitive to detect diastolic dysfunction than conventional echocardiography [9-12]. Despite the relative fluid deficiency after HD, which can induce a decrease of Em and Am, it is very important to evaluate all parameters of LV diastolic function in this population.[6] Apart from LVM and systolic function, the $\mathrm{E} / \mathrm{Em}$ ratio displayed important additional long-term prognostic information [13].

\section{What are the effects of dialysis methods on LV diastolic function?}

Diastolic dysfunction has been observed in patients receiving renal replacement therapy for ESRD in many studies [14-15]. In a study by Duran et al., diastolic function of LV was not significantly altered after maintenance haemodialysis treatment. They demonstrated that in the 
long run, the acute changes of volume, electrolytes and autonomic regulation due to haemodialysis session does not affected left diastolic function. [16] For Lee et al, the prevalence and severity of diastolic LV dysfunction is higher in PD patients [17].

Some authors suggest that LVM and diastolic function are closely related to each other in all dialysis patients [18]. Moreover, in a study by Kimura and al., LVMI was an independent determinant of LV diastolic dysfunction in patients on HD [19]. The prevalence of LVH was higher in conventional HD compared to PD [19]. According to the Framingham criteria, its prevalence was $68.8 \%$ in HD patients and $45.2 \%$ in PD patients [20]. According to that observation, we introduced LVH in our consideration in the evaluation of difference between the two groups concerning LV diastolic function. In fact, in the present study, we observed that the prevalence of $\mathrm{LVH}$ was $44.4 \%$ (40.5\% in HD patients and $52.4 \%$ in PD patients, $p=$ NS). This difference with literature data can be explained by higher age and higher frequency of hypertension in our PD group (although the comparative analysis finds no significant difference concerning those parameters).

However, we introduced severe LVH in a multivariate regression model. We noticed a higher prevalence of diastolic LV dysfunction in the PD group. We also noted that an E/e' $>13$ is the stronger parameter for detection of this LV diastolic dysfunction.

\section{LIMITATION OF OUR STUDY}

Invasive haemodynamics to explore more filling pressure data were not included. The strain and CMR were not performed, that is why we have not been able to explore further the systolic function of the right ventricle.

\section{ETHICAL APPROVAL}

This study was carried out retrospectively; therefore, no ethics committee approval was required. There is no patient identification within this publication. 


\section{DISCLOSURE}

None to declare.

\section{ABBREVIATIONS}

$\mathrm{AVF}=$ ArterioVenous Fistula

$\mathrm{CMR}=$ Cardiovascular Magnetic Resonance

$\mathrm{EF}=$ Ejection Fraction

$\mathrm{ESRD}=$ End Stage Renal Disease

$\mathrm{IVC}=$ Inferior Vena Cava

IVS $=$ Interventricular Septum

$\mathrm{HD}=$ Haemodialysis

GRF $=$ Glomerular Filtration Rate

$\mathrm{LV}=$ Left Ventricle

LVEDD $=$ Left Ventricle End Diastolic Diameter

LVESD $=$ Left Ventricle End Systolic Diameter

$\mathrm{LVM}=$ Left Ventricular Mass

LVPW $=$ Left Ventricular Posterior Wall

LVH $=$ Left Ventricular Hypertrophy

$\mathrm{PD}=$ Peritoneal Dialysis

RAAS $=$ Renin-Angiotensin-Aldosterone System

$\mathrm{RV}=$ Right Ventricle

$\mathrm{RRF}=$ Residual Renal Function

TDI $=$ Tissue Doppler Imaging 


\section{REFERENCES check journal style}

[1] Zoccali C. How important is echocardiography for risk stratification in follow-up of patients with chronic kidney disease? Nature Clin Pract Nephrol 2007; 3:178

[2] Zoccali C, Benedetto FA, Mallafmaci F et al. Prognostic impact of the indexation of left ventricular mass in patients undergoing dialysis. J Am Soc Nephrol 2001;12:2768-74.

[3] Kessler M, Canaud B, Pedrini L et al. Section I. Measurement of renal function, when to refer and when to start dialysis. Nephrol Dial Transplant 2002; 17: 7-9

[4] Ujino K, Barnes ME, Cha SS et al. Two-dimensional echocardiographic methods for assessment of left atrial volume. Am J Cardiol 2006;98:1185-8.

[5] Lang RM, Bierig M, Devereux RB et al. Recommendations for chamber quantification: a report from the American Society of Echocardiography's Guidelines and Standards Committee and the Chamber Quantification Writing Group, developed in conjunction with the European Association of Echocardiography, a branch of the European Society of Cardiology. J Am Soc Echocardiogr 2005;18:1440-63.

[6] Hsiao SH, Lin SK, Huang WC et al. Para-meters Derived from Myocardial Tissue Doppler Imaging Associated with Major Events in Patients with Uremia. Acta Cardiol Sin 2007; 23:254-62.

[7] Kim JK, Kim SG, Kim MG et al. Left Ventricular Diastolic Dysfunction as a Predictor of Rapid Decline of Residual Renal Function in Patients with Peritoneal Dialysis. J Am Soc Echocardiogr 2012, 25: 411-420

[8]Foley RN, Parfrey PS, Harnett JD et al. The prognostic importance of left ventricular geometry in uremic cardiomyopathy. J Am Soc Nephrol 1995; 5:2024-2031.

[9] Hayashi SY, Brodin LA, Alvestrand A, et al. Improvement of cardiac function after haemodialysis. Quantitative evaluation by colour tissue velocity imaging. Nephrol Dial Transplant 2004;19:1497-506. 
[10] Garcia MJ, Thomas JD, Klein AL. New Doppler echocardiographic applications for the study of diastolic function. J Am Coll Cardiol 1998;32:865-75.

[11] Nagueh SF, Middleton KJ, Kopelen HA,et al. Doppler tissue imaging: A noninvasive technique for evaluation of left ventricular relaxation and estimation of filling pressures. $\mathrm{J}$ Am Coll Cardiol 1997;30:1527-33.

[12] Hayashi SY, Rohani M, Lindholm B et al. Left ventricular function in patients with chronic kidney disease evaluated by colour tissue Doppler velocity imaging. Nephrol Dial Transplant 2006;21:125-32.

[13]Wang $\mathrm{AY}^{1}$, Wang M, Lam CW et al . Left ventricular filling pressure by Doppler echocardiography in patients with end-stage renal disease. Hypertension 2008;52(1):107-14. [14]Kimura H, Takeda K, Tsuruya K et al.Left ventricular mass index is an independent determinant of diastolic dysfunction in patients on chronic hemodialysis: A tissue Doppler imaging study. Nephron Clin Pract 2011; 117:c67-c73

[15] Raizada V, Skipper B, Taylor RA et al. Left ventricular diastolic function in patients on hemodialysis. J Investig Med 2010; 58:791-795

[16]Duran M, Unal A, Inanc MT et al. Effect of maintenance hemodialysis on diastolic left ventricular function in end-stage renal disease. Clinics 2010;65:979-84.

[17] Lee J-K et al. Differential association of proinflammatory cytokines with left ventricular diastolic dysfunction in subjects with and without continuous ambulatory peritoneal dialysis. Nutr Metab Cardiovasc Dis 2012;22:974-80.

[18]Weiss G, Lhotta K, Reibnegger G et al. Divergent effects of hemodialysis and continuous ambulatory peritoneal dialysis on cardiac diastolic function. Perit Dial Int 1997;17:353-9. [19]Kimura $\mathrm{H}^{1}$, Takeda $\mathrm{K}$, Tsuruya $\mathrm{K}$ et al.. Left ventricular mass index is an independent determinant of diastolic dysfunction in patients on chronic hemodialysis: a tissue Doppler imaging study. Nephron Clin Pract 2011;117: 67-73. 
[20]Tian JP, Wang T, Wang H et al. The prevalence of left ventricular hypertrophy in Chinese hemodialysis patients is higher than that in peritoneal dialysis patients. Ren Fail 2008;

$30: 391-400$ 


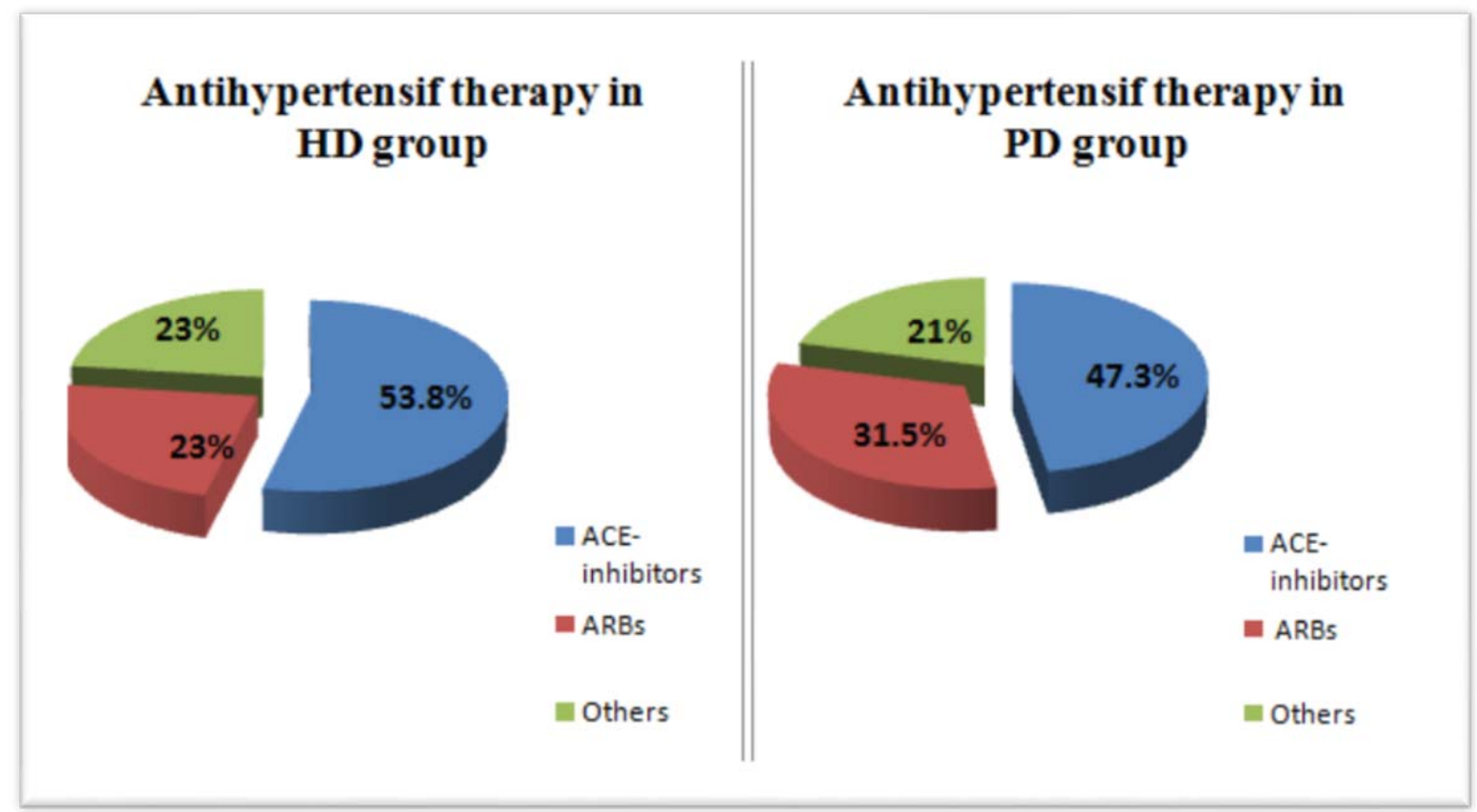

Figure 1: comparison of the use of antihypertensive drugs in the two groups of the study. Spelling of antihypertensive (antihypertensif) within the figure 


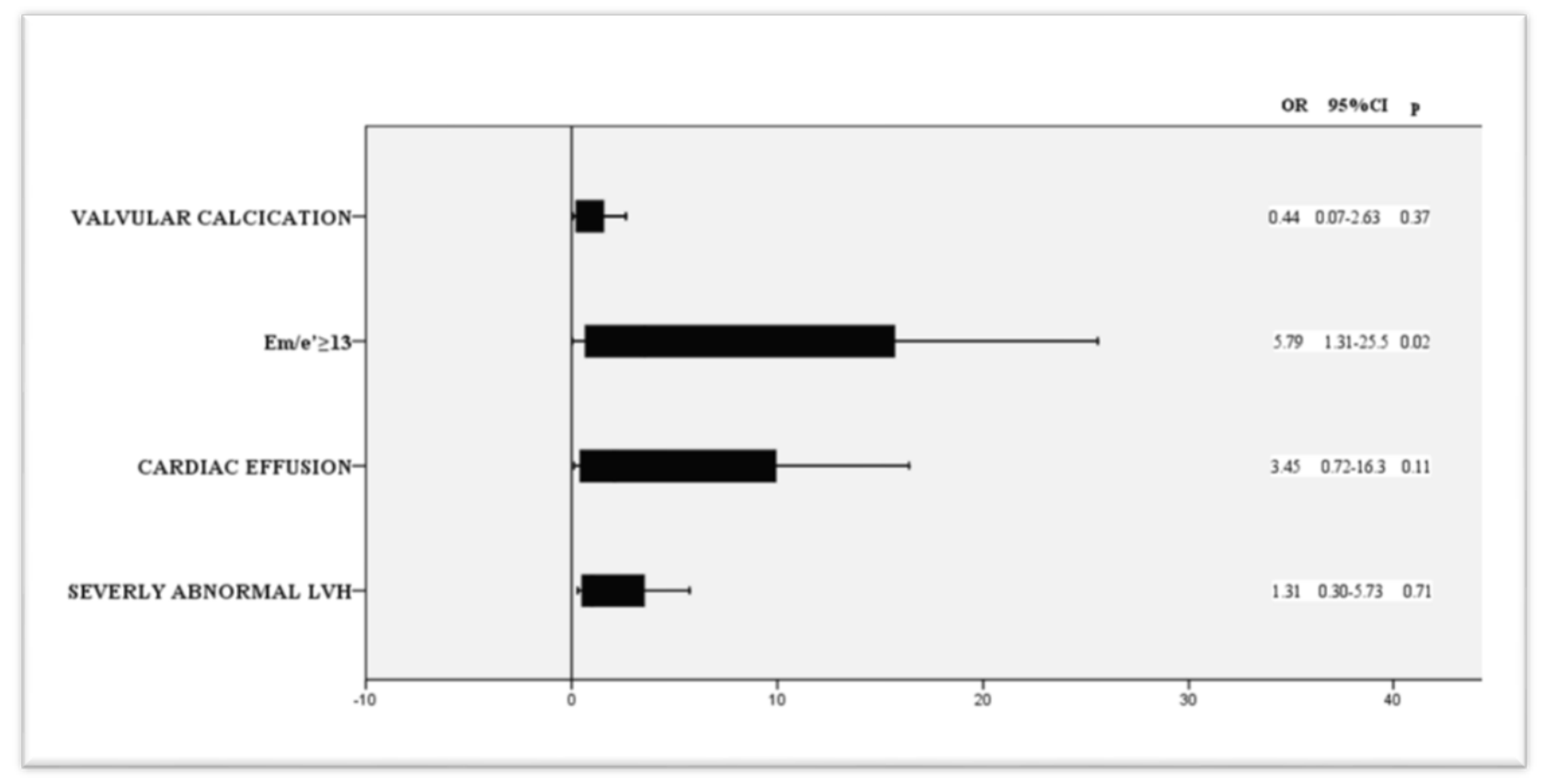

Figure 2: The association between dialysis methods and echocardiographic parameters.

Multiple logistic regression model of statistically significant regressors.

Spelling of calcification in the figure

Table 1: Comparison of Demographic and Biological Characteristics 


\begin{tabular}{|c|c|c|c|}
\hline & HD group & PD group & $p$ \\
\hline & $\mathrm{n}=42$ & $\mathrm{n}=21$ & \\
\hline Male gender $(\%)$ & $21(50)$ & $14(66.6)$ & 0.45 \\
\hline Age (years) & $44.8[33.7-52]$ & $51[39-57.5]$ & 0.13 \\
\hline BMI $\left(\mathrm{Kg} / \mathrm{m}^{2}\right)$ & $22.8 \pm 3.7$ & $22.6 \pm 6.8$ & 0.57 \\
\hline Weight (kg) & $65.2 \pm 14.2$ & $67.5 \pm 14.5$ & 0.39 \\
\hline Hypertension (\%) & $18(45)$ & $13(61.9)$ & 0.21 \\
\hline Dialyse duration (years) & $3[2-6]$ & $3[2-4.5]$ & 0.07 \\
\hline $\operatorname{GFR}\left(\mathrm{mL} / \mathrm{min} / 1.73 \mathrm{~m}^{2}\right)$ & $5.1[2.8-6.07]$ & $4.9[3.9-5.8]$ & 0.9 \\
\hline Triglycerides (mmol/1) & $1.1[0.8-1.5]$ & $1.3[0.9-1.8]$ & 0.45 \\
\hline LDL-C (mmol/l) & $1.1[0.9-1.27]$ & $1.1[0.9-1.4]$ & 0.7 \\
\hline CRP (mg/l) & $4[2.3-6]$ & $4[2-6]$ & 0.6 \\
\hline Uric acid (mg/l) & $61[55-76]$ & $72[60-80]$ & 0.08 \\
\hline \multirow{3}{*}{$\begin{array}{l}\text { Calcemia what does this } \\
\text { mean? Units? }\end{array}$} & $90 \pm 8.6$ & $86 \pm 8.8$ & 0.12 \\
\hline & $47[28-65]$ & $49.8[44-61.5]$ & 0.051 \\
\hline & $4(9.5)$ & $3(14.2)$ & 0.7 \\
\hline $\begin{array}{l}\text { Phosphoremia what does } \\
\text { this mean? Units? }\end{array}$ & $5(12)$ & $4(19)$ & 0.7 \\
\hline \multicolumn{4}{|l|}{ Anaemia (\%) } \\
\hline Smoking (\%) & & & \\
\hline
\end{tabular}

BMI: Body Mass Index; LDL-C: low-density lipoprotein cholesterol; GFR: CRP: 
Table 3: Comparison of Comparison of the two-dimensional and M-mode findings

\begin{tabular}{lccc}
\hline & HD Group & PD Group & $p$ \\
& $\mathrm{n}=42$ & $\mathrm{n}=21$ & \\
\hline LVEDD $(\mathrm{mm})$ & $51.2 \pm 5.6$ & $51.2 \pm 8.9$ & 0.41 \\
LVEDD index $\left(\mathrm{mm} / \mathrm{m}^{2}\right)$ & $30.1 \pm 4$ & $30 \pm 4.9$ & 0.33 \\
LVESD (mm) & $32.1 \pm 8$ & $30.2 \pm 4.9$ & 0.18 \\
LVESD index $\left(\mathrm{mm} / \mathrm{m}^{2}\right)$ & $19.2 \pm 4.7$ & $18.2 \pm 2.8$ & 0.11 \\
IVS $(\mathrm{mm})$ & $9.6 \pm 2.6$ & $10.6 \pm 2.6$ & 0.07 \\
PW (mm) & $9.7 \pm 2.3$ & $10.3 \pm 2$ & 0.2 \\
RWT & $0.38 \pm 0.09$ & $0.41 \pm 0.1$ & 0.18 \\
LVM & $174[131-220]$ & $210[150-242]$ & 0.19 \\
LVM index & $124[88-170]$ & $108[76-141]$ & 0.14 \\
LVH (n $(\%))$ & $17(40.5 \%)$ & $11(52.4 \%)$ & 0.45 \\
Severely abnormal LVH & $\mathbf{1 0}(\mathbf{2 3 . 8 \% )}$ & $\mathbf{9 ( 4 2 . 9 \% )}$ & $\mathbf{0 . 0 2}$ \\
EF $(\%)$ & $64.3 \pm 9.7$ & $64.7 \pm 12.3$ & 0.38 \\
RVEDD (mm) & $24 \pm 4.4$ & $22.2 \pm 3.9$ & 0.13 \\
TAPSE $(\mathrm{mm})$ & $23.3 \pm 3.4$ & $24.4 \pm 3.8$ & 0.52 \\
RVS (mm) & $14.3 \pm 2.2$ & $15 \pm 1.4$ & 0.36 \\
IVC diameter (mm) & $9[6-11]$ & $10.6[7.5-13.5]$ & 0.05 \\
RA Area (cm $\left.{ }^{2}\right)$ & $16.7 \pm 7.9$ & $13.8 \pm 2.7$ & 0.77 \\
LA Area (cm $\left.{ }^{2}\right)$ & $17.8 \pm 6.6$ & $18.4 \pm 3.9$ & 0.44 \\
LA volume (ml) & $44[33-77.5]$ & $57[39-72]$ & 0.78 \\
PASP & $30.8 \pm 9.9$ & $31 \pm 7.8$ & 0.81 \\
Valvular calcification & $\mathbf{9 ( 2 1 . 4 \% )}$ & $\mathbf{2 ( 9 . 5 \% )}$ & $\mathbf{0 . 0 0 1}$ \\
\hline
\end{tabular}

LVEDD: left ventricular end-diastolic diameter; LVESD: left ventricular end-systolic

diameter; IVS: inter ventricular septum; EF: Ejection fraction; RVEDD: right ventricular end-

diastolic diameter; RV: right ventricle; S: peak systolic velocity; e': peak early diastolic

velocity; PW: posterior wall; TAPSE: tricuspid anteroposterior systolic excursion; IVC:

inferior vena cava; LA: Left atrial; RA: Right atrial make sure you add units to every

variable 
Table 4: Comparison of filling pressures features between the two groups

\begin{tabular}{lccc}
\hline & HD Group & PD Group & $p$ \\
& $\mathrm{n}=42$ & $\mathrm{n}=21$ & \\
\hline $\mathrm{Em} / \mathrm{Am} \geq 2$ & $0(0 \%)$ & $1(4.7 \%)$ & 0.09 \\
Em/e' $\geq \mathbf{1 3}$ & $\mathbf{5 ( 1 1 . 9 \% )}$ & $\mathbf{8 ( 3 8 . 1 \% )}$ & $\mathbf{0 . 0 0 4}$ \\
PASP $>35 \mathrm{mmHg}$ & $5(11.9 \%)$ & $1(4.7 \%)$ & 0.55 \\
Pericardial effusion & $\mathbf{5 ( 1 1 . 9 \% )}$ & $\mathbf{1 0}(\mathbf{4 7 . 6 \% )}$ & $\mathbf{0 . 0 0 3}$ \\
LA volume $>52 \mathrm{ml}$ & $15(35.7 \%)$ & $7(33.3 \%)$ & 0.36 \\
\hline
\end{tabular}

Em: Early diastolic wave; Am: Atrial wave; e': peak early diastolic velocity; PASP:

pulmonary artery systolic pressure 